\title{
Fast Region of Interest Detection for Fetal Genital Organs in B-mode Ultrasound Images
}

\author{
R Bharath, P Rajalakshmi, \\ Department of Electrical Engineering, \\ Indian Institute of Technology, Hyderebad, \\ email:\{ ee13p0007, raji \}@iith.ac.in
}

\begin{abstract}
Genital organ detection of fetus in B-mode ultrasound images has a considerable significance. It is useful to know any malformations present in the genital organs and also to determine the sex of the fetus. In this paper we propose a Feature from Accelerated Segment Test (FAST) technique for approximate detection of fetal genitals in ultrasound images. FAST algorithm is capable of producing the corner points at a higher speed which falls on the fetal genital organs. A window of size $60 \times 60$ pixels being corner point as a center is considered as Region of Interest (ROI), where genital organ of fetus is anticipated. The efficiency of the algorithm is calculated as the ratio of number of images where corner points are placed on the fetus genital organ to the total number of images tested. FAST algorithm is robust to speckles present in the image, machine independent, fast and also computationally less intensive to implement in real time with an efficiency of 96.7\%
\end{abstract}

Index Terms-Fetus Genital organ, prenatal sex discernment, ultrasound scanning, Corner detection, FAST algorithm

\section{INTRODUCTION}

Medical Ultrasound Imaging is the safest modality used to monitor health condition of a fetus. Genital organ detection in B-mode ultrasound images is done to know the malformations of genital organs. $95 \%$ of prenatal sex discernment is also done through ultrasound scanning. Unlikely, which also leads to fetal feticide causing worsen gender imbalance [1] in most of the Asian countries including India, China etc. Prenatal sex discernment followed by prenatal abortions still continues despite the strong amendments made by the governments [2]. Automatic fetal genital detection will help to mask the genital organs of fetus not revealing the sex of a fetus preventing from prenatal sex discernment, if needed.

Ultrasound Imaging is a real time modality where sonographers have to make decisions from real time streaming videos and still images which is feeezed from this videos. Detecting organs in ultrasound images is a challenging task both for machine and sonographer due to quality of image produced by ultrasound system are very poor. Sonographer has to look for entire image for detecting small organs like genitals, stones etc, which is time consuming. In this paper, we come up with computer vision algorithm to detect the approximate location of the organ which will drastically reduce the search space for the organ detection in the ultrasound image.

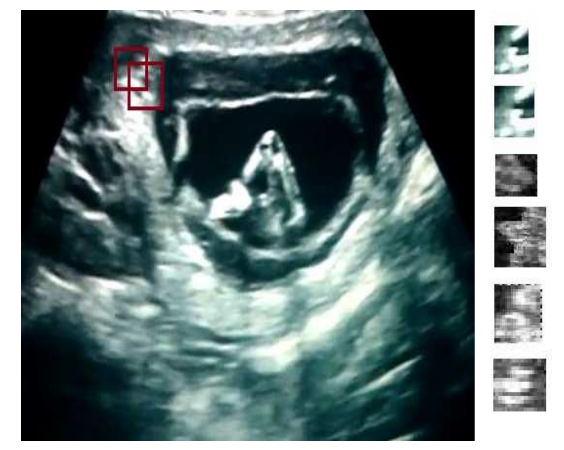

Fig. 1: Template Matching.

Ultrasound fetal genital organ detection is strongly influenced by quality of data. The characteristic artefacts such as speckles, attenuations, signal dropouts, nonlinear operation of gain (Time Gain Compensators, Gain) and missing boundaries of organs make detection algorithm complicated. Addition to above said artefacts the genital organs differ in shape from male to female, size of the organ varies depending upon the gestational age, does not have closed boundaries and shape of the organ varies with the field of view of an ultrasound probe.

Despite all these artefacts, the anatomy of fetus in womb itself gives some useful information to take advantage for fast automatic detection of genitals of fetus. The amniotic fluid present around the genitals represent low intensity region providing sharp contrast to genital organs in B-mode ultrasound images. The genital organs are curvy and smooth in nature. Independent organs will not present near the genital organs. The genital organs lie on the edges of the ultrasound image unless occluded with thighs. These are desirable features which ensure the presence of corner points on the genital organ. Corner points are considered as Pixel of Interest (POI) in this context.

Fetus sex is detected by visualizing the genital tubercle direction. Downward direction of genital tubercle indicates female fetus while upward direction indicates 


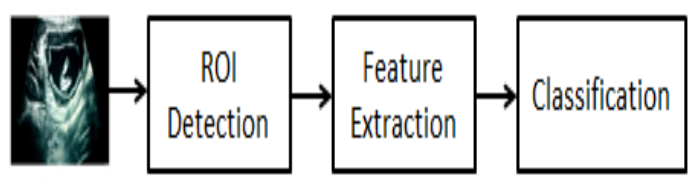

Fig. 2: Block diagram of Automatic organ detection.

male fetus. Male fetus is also recognized by seeing other landmarks such as fetal scrotum or penis. Female fetus is identified by seeing dome shaped structure or three lines. These landmarks are visible in the ultrasound images [3].

The automatic organ detection is normally done using a supervised classifier or by template matching method. In template matching method the genital ultrasound image template is placed at every possible location in fetus ultrasound image and matching performance is found for each location, if good match is found then we confirm that organ is present in that particular location. The matching measure can be normalized cross correlation, sum of squared distance, sum of absolute difference and maximum difference. Template matching does not work when the organ is skewed, scaled or rotated. To overcome this, we have to take possible transformations of each female and male genital organ as templates and have to find the matching performance of each template as shown in the Fig. 1. Right side of the image represents some of the organ transformation templates used as query templates to detect the genital organ in the test image. Red rectangular boxes indicate the possible locations in the test image where matching measures are done.

Automatic genital organ detection can also be done with a supervised classifier which is trained with rotational, position, texture, shape and size invariant features [4], [5], [6], [7], and [8] of genital and non-genital organs of an ultrasound image. The supervised classifier will give the confidence measure of each window, based on the confidence measure of each window, we confirm the presence of genital organs in the image. These recognition algorithms are generally based on sliding window model technique, where the test image is divided into overlapping windows and detection is done at every window. Genital organ detection in sliding window model suffers from high computations as matching criteria has to be done in every possible location in the test image. Implementation of sliding window model is possible but not feasible in real time image processing applications.

Fig. 2 gives the block diagram representation of automatic organ detection. In this paper we address how to detect ROI for genital organs in the image such that matching criterion is done only at those positions, reducing the search space in the image. This will improve the speed of detecting genital organs in the ultrasound image.
The rest of the paper is organized in the following way. Section II gives the idea of how to detect the corner points on the genitals. In Section III, we discuss adaptability and working of FAST technique to find the corner points. Results in terms of efficiency and fastness of the technique are discussed in Section IV. Section V concludes the paper.

\section{Algorithm FRAMEWORK}

To overcome the problem of finding matching measure at every possible location, [9] proposed a method based on curvature of a genital region as an underling feature to detect the ROI of genital organ. In this method corner points on the genital organ are found by first detecting the contour of the image and sampling the contour of the image appropriately to resemble the shape of the genital organs. If curvature of the sampled contour points crosses the fixed threshold then it is considered as corner points, an appropriate window being corner point as center is considered as ROI. Contour detection of ultrasound image without any preprocessing of the image using canny operator is shown in Fig. 3. We observe granularity and snake like structures in the image which actually not correspond to actual scene of the image. These are occurred due to speckle noise present in the ultrasound images. Formation of speckles in ultrasound images is explained in [10]. Sampling and curvature detection of sampled contour points using canny operator produces the corner points all over the image which is not required.

Contour detection of ultrasound image is not straight forward as it involves a series of steps as shown in Algorithm 1. Ultrasound images do not have sharp boundaries which are affected due to speckles [11]. Speckles cause the delineation of edges in ultrasound images. In step 1, speckle suppression filters [12] are used to suppress the speckles in the image. In step 2, the image is binarized to get the segmented image. Genital organs are not isolated, so isolated objects whose area is less than some fixed threshold are removed in step 3. If we do not remove the isolated objects then probability

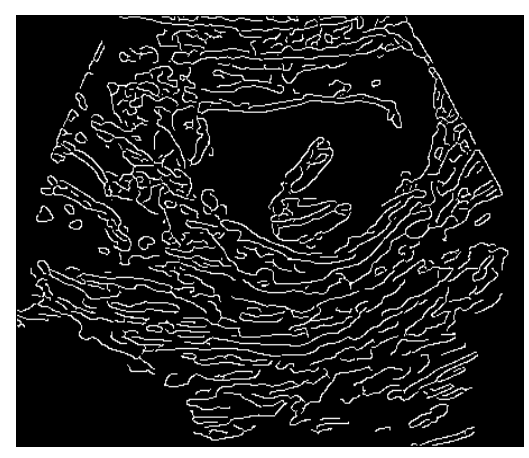

Fig. 3: Contour detection without preprocessing. 


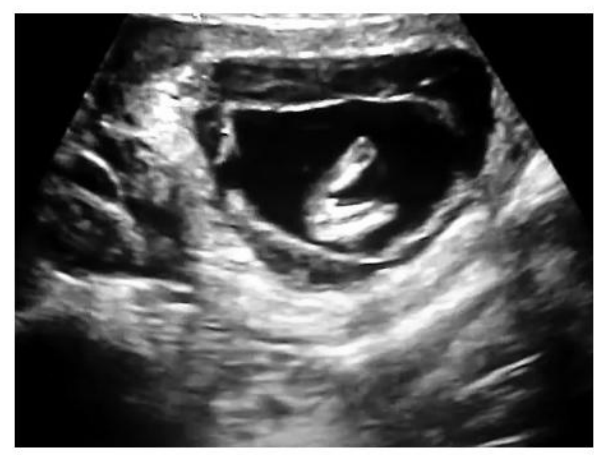

(a) Ultrasound Image.

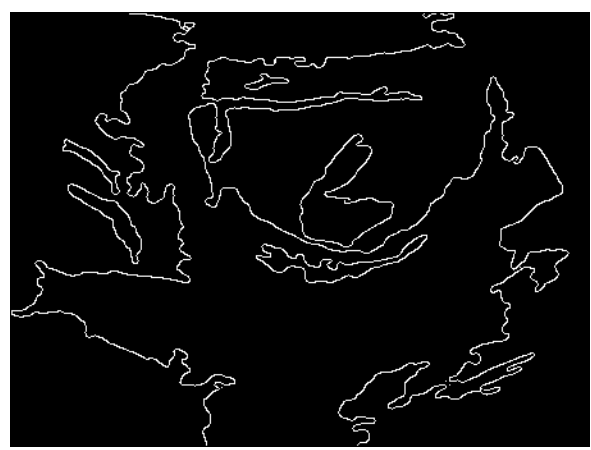

(c) Contour of the segmented image.

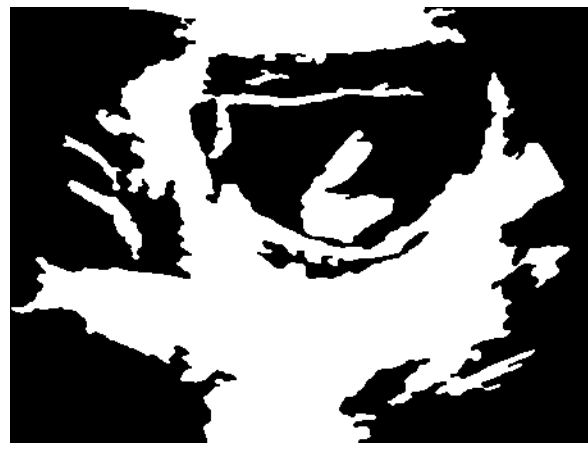

(b) Segmented Image.

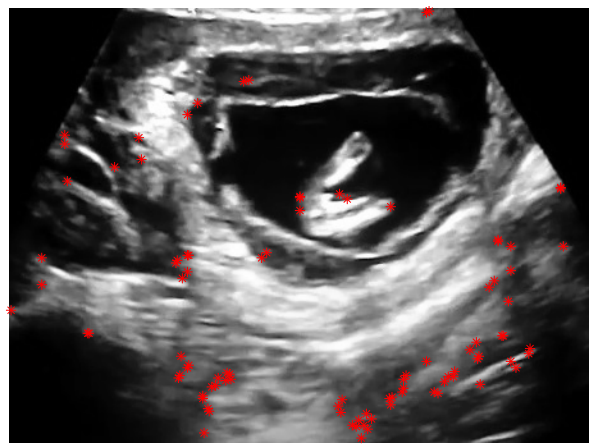

(d) POI on the image.

Fig. 4: Detection of POI using Curvature Method.

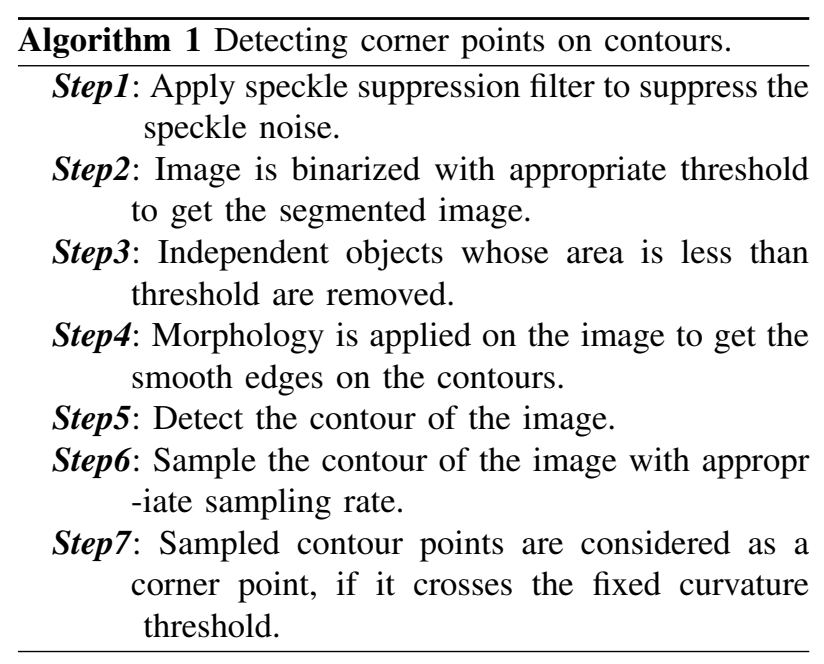

of getting corner points on the isolated contours will be higher. The effects of textures and speckles makes the image to have nonsmooth contours, but the organs are always smooth, to obtain smoothness on the boundaries, image morphology imclose operator [13] is applied on the image in step 4. In step 5, edges of the morphed image are detected using canny operator. In step 6, edges are sampled appropriately to resemble the shape of the organs. In step 7, the curvature of each sampled contour point is computed and considered as a corner point only if it crosses some fixed threshold.

The curvature of each sampled contour point $\left(x_{i}, y_{i}\right)$ with respect to $\left(x_{i-1}, y_{i-1}\right),\left(x_{i+1}, y_{i+1}\right)$ is computed in the following way [14].

$$
\begin{gathered}
\Delta x_{i}=\frac{x_{i+1}-x_{i-1}}{\sqrt{\left(x_{i+1}-x_{i-1}\right)^{2}+\left(y_{i+1}-y_{i-1}\right)^{2}}} \\
\Delta y_{i}=\frac{y_{i+1}-y_{i-1}}{\sqrt{\left(x_{i+1}-x_{i-1}\right)^{2}+\left(y_{i+1}-y_{i-1}\right)^{2}}} \\
\Delta^{2} x_{i}=\frac{\frac{x_{i+1}-x_{i}}{\sqrt{\left(x_{i+1}-x_{i}\right)^{2}+\left(y_{i+1}-y_{i}\right)^{2}}}-\frac{x_{i}-x_{i-1}}{0.5 \sqrt{\left(x_{i+1}-x_{i-1}\right)^{2}+\left(y_{i+1}-y_{i-1}\right)^{2}}}}{\Delta^{2} y_{i}=\frac{\frac{y_{i-1} x_{i-1}}{\sqrt{\left(x_{i+1}-x_{i}\right)^{2}+\left(y_{i+1}-y_{i}\right)^{2}}}-\frac{y_{i}-y_{i-1}}{\sqrt{\left(x_{i}-x_{i-1}\right)^{2}+\left(y_{i}-y_{i-1}\right)^{2}}}}{0.5 \sqrt{\left(x_{i+1}-x_{i-1}\right)^{2}+\left(y_{i+1}-y_{i-1}\right)^{2}}}}
\end{gathered}
$$

here $\Delta$ and $\Delta^{2}$ denotes the first order and second order 
difference operators.

Curvature at that point $\left(x_{i}, y_{i}\right)$ is given by

$$
\text { curv }=\Delta x_{i} \Delta^{2} y_{i}-\Delta y_{i} \Delta^{2} x_{i}
$$

$\left(x_{i-1}, y_{i-1}\right),\left(x_{i}, y_{i}\right)$ and $\left(x_{i+1}, y_{i+1}\right)$ represents the consecutive spatial coordinates of the sampled contours of the image. The high magnitudes of curvature values resulted due to small magnitudes of euclidean distance between $\left(x_{i-1}, y_{i-1}\right),\left(x_{i+1}, y_{i+1}\right)$. The results of Algorithm 1 are shown in Fig. 4. The B-mode ultrasound image having male fetal is shown in Fig. 4(a), Segmented image after removing independent objects is shown in Fig. 4(b), Contour of the segmented image is shown in Fig. 4(c), POI in B-mode ultrasound image are shown with red colored ' $*$ ' marks is shown in Fig. 4(d).

To improve the efficiency of the algorithm we have to reduce the curvature threshold which inturn increases the number of corner points which crosses the fixed threshold. This will improve the efficiency of the algorithm by ensuring the presence of corner points on the genital organ. More number of corner points emerged from buttocks, foot contours of fetus and boundaries of womb which will have considerable curvatures compared to genital organ curvature. Increase in number of corner points will reduce the speed of detection of genital organ as it varies inversely to number of corner points produced on the image. If we fix high curvature threshold, then the probability of getting corner points on genital organs are reduced, this will increase the percentage of false negatives in the detection algorithm as detection is done only for ROI corresponding to corner points.

This way of finding POI on genitals involves more computations as it involves a lot of preprocessing. In this paper we propose to use Feature from Accelearted Segment test (FAST) algorithm, which is applied directly on the ultrasound images and also effective in placing POI on the genital organs.

\section{FAST ALGORITHM FOR FINDING CORNER POINTS ON THE FETAL GENITAL ORGANS}

Corner point detection using FAST algorithm [15] depends on the relative brightness of the neighbouring pixels. The computer vision of genital organ is shown in Fig. 5. The values inside the boxes represents the pixel brightness intensities. The corner points detection using FAST algorithm technique is explained in Algorithm 2, taking Fig. 5 as reference.

The amniotic fluid present around the fetus will help the FAST algorithm to place the corners on genitals much more efficiently. Amniotic fluid corresponds to dark pixels and a fetus genital corresponds to bright pixels in ultrasound images. When we consider a pixel on genital, there is a high probability of crossing ' $\mathrm{m}$ ' contiguous pixels having ' $I_{k}-t$ ' intensities; these ' $I_{k}-t$ ' intensities will arise mostly from the amniotic fluid

\begin{tabular}{l}
\hline Algorithm 2 Corner point detection using FAST \\
algorithm. \\
\hline Step 1: Consider a pixel in the image say K which \\
is to be identified as POI or not. Let the \\
intensity of the pixel be $I_{k}$. It is shown in \\
thick black rectangular box in Fig. 5 and \\
here its value is 144 .
\end{tabular}

Step2: Fix some appropriate threshold value $I_{t}$. Here $I_{t}$ is chosen as 20 .

Step3: Form a circle of 16 pixels around the POI. These pixels are shown in gray shaded boxes.

Step4: The pixel K is considered as a POI, if the circle consists of a set of $\mathrm{m}$ contiguous pixels which are brighter than $I_{k}+I_{t}$ or all darker than $I_{k}-I_{t}$. (Here $\mathrm{m}$ is chosen to be 12 , shown in thin black line. )

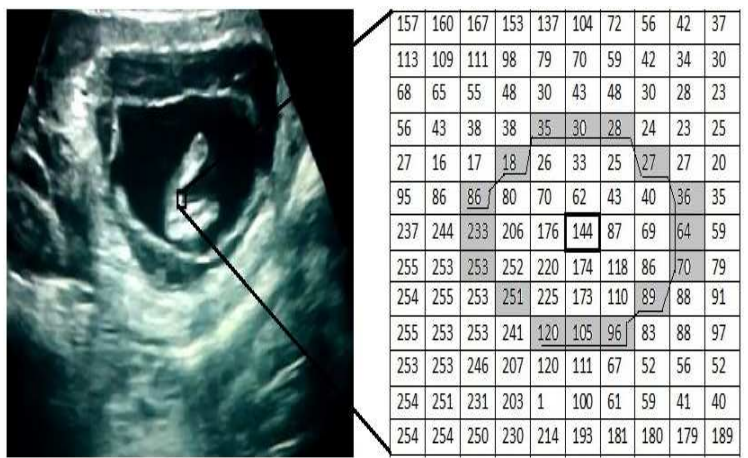

Fig. 5: Computer vision of genital organ.

region. The amniotic fluid will provide the required contrast to the genital organs irrespective of variation of gain knobs of ultrasound machine making this algorithm robust to variable gain of the ultrasound machine. The strength of corner points are determined by the summation of the absolute differences of corner point pixel intensity to $\mathrm{m}$ contiguous pixel intensities in the circle, shown with black line in gray shade region in Fig. 5. The parameters $I_{t}, \mathrm{~m}$ and number of corner points are selected by validating the performance of algorithm by changing these parameters. The optimum performance is obtained when $I_{t}, \mathrm{~m}$ is equal to 20,12 respectively.

\section{RESULTS}

FAST algorithm is applied on thirty fetus ultrasound images consisting of 20 male and 10 female fetus of different gestational ages. In twenty nine images FAST algorithm placed POI on fetus genitals getting an overall efficiency of $96.7 \%$ which is better than curvature detection algorithm with an efficiency of $95 \%$ [9]. FAST algorithm is failed in one image in which genital organ is partially occluded by thighs of the fetus. FAST algorithm is tested on the images acquired from the ultrasound machines with variable gains. These 


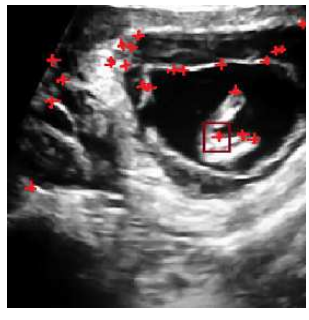

(a)

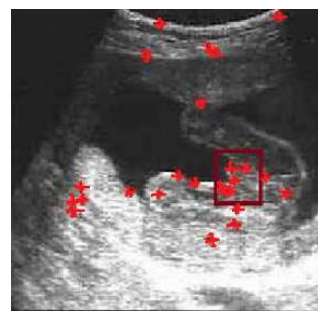

(c)

(d)
Fig. 6: Detection of POI using FAST Method.

ultrasound images had manufacturer logos, which had to be cropped manually. Algorithms are implemented in MATLAB R2013a using Intel CORE i5 processor with 8GB RAM. FAST algorithm took $81.5 \mathrm{~ms}$ for computing the corner points of $360 * 320$ pixel image.

In FAST algorithm, twenty five strongest corner points are sufficient to place the corner points on the genital organ. Fig. 6(a), 6(b), 6(c) and 6(d) represents outcome of FAST algorithm for some of the sample images from database. The genital organs are shown inside a rectangular box and corner points with red colored '*' marks. An appropriate window of size $60 \times 60$ around the POI is considered as ROI where fetal genital organ expected to present. 60x60 window around POI is selected as it covers both foreground (genital organ) and background (Amniotic fluid, thighs and buttocks), features from this ROI will give good descriptors of the organ. If two POI lies on side by side then one POI is dropped as ROI overlaps for both the POI.

\section{CONCLUSION}

FAST algorithm is applied directly on ultrasound images without any need for preprocessing which reduces the computational overhead to implement this algorithm in real time image processing. This algorithm is robust to speckles and machine dependent parameters which includes TGC, gain knobs etc, as it looks into a set of predefined neighbours which is local to that pixel of interest. The obtained results shows that FAST algorithm is capable of placing POI on genitals with an efficiency of $96.7 \%$ with less computations.
We are working on extraction of efficient features from ROI and suitable classifier to detect the genital organs in the B-mode ultrasound images.

\section{REFERENCES}

[1] B. R. Sharma, N. Gupta and N. Relhan, "Misuse of prenatal diagnostic technology for sex-selected abortions and its consequences in India," Journal Royal Inst. Pub. Health,vol.121, pp.854-860, 2007.

[2] Amendment in India, Available online at http://pndt.gov.in/writereaddata/mainlinkFile/File50.pdf.

[3] Yudha Noor Aditya, Heamn Noori Abduljabbar, Christina Pahl, Lai Khin Wee, Eko Supriyanto, "Fetal Weight and Gender Estimation using Computer based Ultrasound Images Analysis,"International Journal of computers, vol.1.7, pp.11-21, 2013.

[4] Haralick, R., Shanmugam, K., and Dinstein, I,

"Textural Features for Image Classification",IEEE Transactions on Systems, Man, and Cybernetics, SMC-3, vol.6, pp. 610621, 1973.

[5] Khotanzad, A., and Hong, Y, "Invariant image recognition by Zernike moments",Pattern Analysis and Machine Intelligence, IEEE Transactions, vol.12.5, pp.489-497, 1990.

[6] Dufour,E.L;Miller, E.L.Galatsanos, "N.P Template matching based object recognition with unknown geometric parameters",Image Processing, IEEE Transactions, vol.11.12, pp.13851396, 2002.

[7] Dalal, N., and Triggs. H., "Histograms of Oriented Gradients for Human Detection",CVPR,IEEE computer society conference, vol.1, pp. 886-893, 2005.

[8] Ojala, T., Pietikainen, M., and Maenpaa, T., "Multiresolution gray-scale and rotation invariant texture classification with local binary patterns",Pattern Analysis and Machine Intelligence, IEEE Transactions, vol.24.7, pp.971-987, 2002.

[9] Sheng TANG, SI-ping CHEN "A fast automatic recognition and location algorithm for fetal genital organs in ultrasound" Journal of Zhejiang University SCIENCE B, pp.1862-1783 (Online), 2009.

[10] J. W. Goodman, Statistical properties of laser speckle patterns, in Laser Speckle and Related Phenomena, J. C. Dainty, Ed. Berlin, Germany: Springer-Verlag, pp. 975,1984.

[11] R.F.Wagner, S.W.Smith, H.Lopez, "Statistics of speckles in ultrasound B-scans", Sonics and Ultrasonics, IEEE Transactions, vol.30.3, pp.156-163, 1983.

[12] Karaman, M., Kutay, M., Bozdagi, G., "An adaptive speckle suppression filter for medical ultrasonic imaging,". IEEE Trans. Med. Imaging, vol.14.2, pp.283-292, 1995.

[13] R.C.Gonzalez, R.E. Woods, Digital Image Processing, Pearson Prentice Hall, Upper Saddle River, New Jersey, 2008.

[14] Rattarangsi A. and R.T. Chin,"Scale-based detection of corners of planar curves,"IEEE Transactions on Pattern Analysis and Machine Intelligence, vol. PAMI-14, pp. 430449, 1992.

[15] Edward Rosten and Tom Drummond, "Machine learning for high speed corner detection",European Conference on Computer Vision,vol.1, pp.430-443, 2006. 